\section{Learning and recall differentiated in serial learning'}

STEPHEN C. WOODS and MICHAEL $L$. EPSTEIN, University of Washington, Seattle, Wash. 98105

In a serial learning experiment in which the first item was made unique, the controls learned the list in the usual manner. For the experimental group, each trial began at a different, randomly chosen syllable. The results show that (a) acquisition is impaired and there is a different curve for the experimental group; $(b)$ the unique item has an anchoring effect; and (c) a plot of errors within each trial shows no serial position effect (SPE) in the experimental group. The results are interpreted to indicate that: (a) anchoring accounts for a substantial portion of the SPE, and (b) recall shows no $S P E$ in this paradigm.

The serial position effect (SPE) is one of the most robust phenomena in verbal learning. The SPE is reliable enough to be observed in individual Ss as well as in group data and occurs during both learning and items in a fixed order results in a bowed curve with fewest errors per item at either end of the list and more errors towards the middle. More precisely, most errors usually occur on an item slightly after the center of the list. The curve, therefore, is skewed to the left, so that in a 12-item list, most errors

In the usual procedure, a list of items is presented one by one in a fixed order and at a constant rate. $S$ attempts to anticipate each successive item. An intertrial interval typically exists between the presentation of the last item in one trial and the first item in the next trial. The trials are repeated with the items in the same order until S learns the list to some criterion.

Many alterations have been made in this classical technique, but they have resulted in little or no change in the shape of the serial learning curve. The list has been presented with no intertrial interval (Glanzer \& Kresse, 1952), and with varying starting items but the same order on each trial (Bowman \& Thurlow, 1963). None of these alterations has significantly altered the shape of the serial learning curve. Results from a study by Glanzer \& Peters (1962) led them to conclude that neither changes in spacing and starting position nor the addition of association breaks (i.e., gaps following every two items) affected the shape of the learning curve. Similarly, it appears that any method which results in increased efficiency in recall (Slamecka, 1967). Learning a list of occur on about the 8 th item. learning a serial list reduces the absolute errors to criterion without changing the basic shape of the serial learning curve (McCrary \& Hunter, 1953).

Even though the SPE has displayed great robustness, we feel that certain aspects of the phenomenon remain unexplained. This experiment shows that under these particular conditions, (1) a minimum of one-half of the SPE can be attributed to anchoring effects associated with the intertrial interval, and (2) the processes that cause the SPE are associated with learning rather than recall. METHOD

The Ss were 20 female undergraduates who participated to fulfill part of the requirements of an introductory psychology class. Ss were randomly divided into two groups. The 10 control Ss were presented a list of 15 nonsense syllables of 46-53\% association (Glaze, 1928) on a memory drum. Each syllable was presented for $3 \mathrm{sec}$ in the standard anticipation method, and the intertrial interval was $3 \mathrm{sec}$. During the intertrial interval, three asterisks were visible in the window of the memory drum. The syllable designated as the first item in the list was distinguished from the rest by the presence of a numeral, " 1 ," to the left of it. Ss were presented the list in serial order until a criterion of one errorless trial was reached, or until 30 trials were completed.

The 10 experimental Ss were presented the same serial list in the same manner on the first trial; the numeral "l "labelled the same syllable. However, on all subsequent trials, the item on which any trial began was chosen at random. Then the entire list was presented in sequential fashion, ending with the item that normally preceded the first item chosen on that particular trial. For example, if a particular trial started with the seventh item in the list (six syllables af ter the one labelled with the numeral " 1 "), the entire list followed it in sequence, the trial ending with the fifth item after the labelled item. Item 1 followed Item 15 without a break, and hence, Item 15 soon became a cue for Item 1 . This essentially resulted in a the numeral " 1 " serving as the only unique item. The same criterion was used for this group was used for the controls. If a given $S$ required 30 trials, each syllable served twice as the starting point for a trial.

Errors on each syllable in each trial were recorded

The results are shown in Fig. 1. The actual data points as well as the best-fitting curves are plotted. The control group demonstrated the classic SPE, as described by Hovland (1938). The extremes of the list were easier to learn than the center, and the most difficult items were those just past the center of the list. The experimental group also showed greater ease of learning at the extremes than in the center, and a similar skew was obtained. The maxima for the two curves lie between Items 8 and 9, i.e., 8.29 for the experimental group and 8.56 for the control group. These are not significantly different. It should be noted that for the experimental group the first item is plotted circular list with the syllable labelled with

\section{RESULTS}

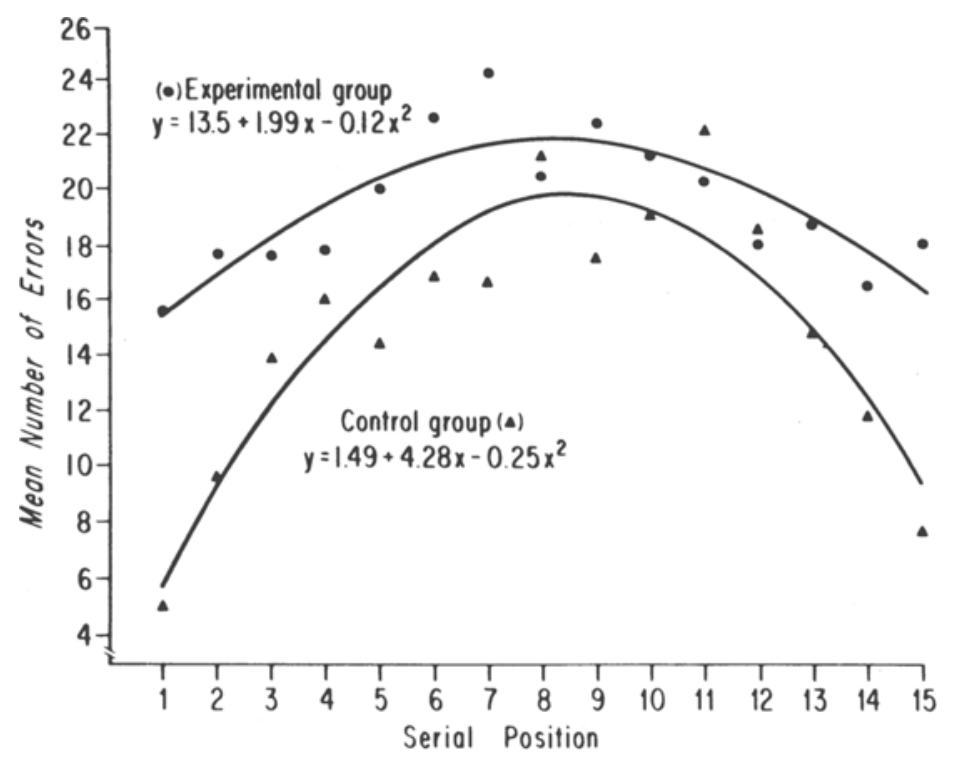

Fig. 1. The best fitting curves and actual data points for the two groups. Mean errors are plotted against serial position. 


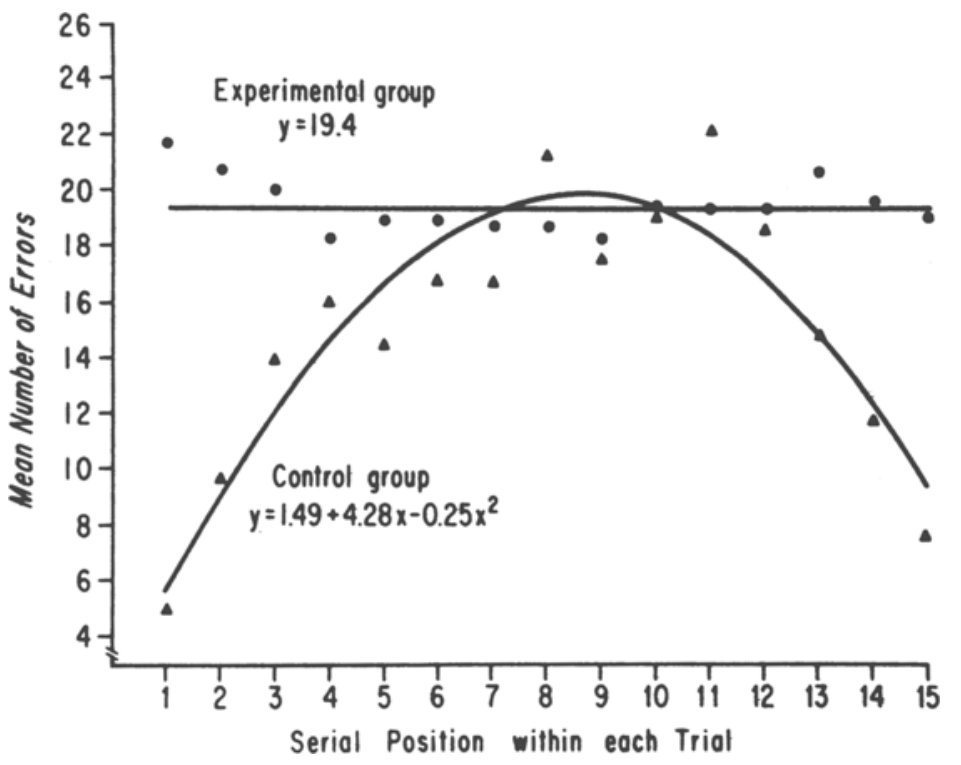

Fig. 2. The best fitting curves and actual data points of the two groups when mean errors are plotted against serial position within each trial. The experimental curve is drawn as a horizontal line because it had neither a significant quadratic nor linear trend.

at the end of the list for reasons to be discussed below. Both curves individually have significant quadratic trends (control: $F=109, p<.01$; experimental: $F=19.3$, $\mathrm{p}<.01$ ), and there is a significant difference between the quadratic trends of the two groups $(F=15.9, p<.01)$. Each group also has a significant linear trend, but there is no significant difference between the linear trends $(\mathrm{F}=2.55, \mathrm{p}>.05)$. There is also a significant difference between the total number of errors made by the two groups $(\mathrm{F}=25.7, \mathrm{p}<.01)$.

Figure 2 shows the errors for each group, plotted against serial position within each trial. The control group curve is the same as it is in Fig. 1 since serial position, and serial position within each trial, are the same for this group. The experimental group showed neither a significant linear nor quadratic trend when analyzed in this way and hence is shown as a straight line with no vertical deviations. Both the linear and quadratic trends of the experimental group are significantly different from those of the control group.

\section{DISCUSSION}

One of the most interesting findings is that the serial curve is bowed when the initial item for each trial is randomly selected. As is shown in Fig. 1, both the experimental and control groups make fewer errors at the beginning and end of the list (as defined by the item with the numeral " 1 ") than in the center. Although the quadratic trends of the two curves differ significantly, the points where they are maximum do not. This reflects the robustness of the skew in the SPE. The difference in quadratic trends is probably due to the fact that more $S s$ in the experimental group required 30 trials than Ss in the control group, i.e., there is a ceiling here. Bowman \& Thurlow (1963), who also plotted their data in this manner, found no difference between the quadratic trends of the two groups. The 12-sec intertrial interval of their experimental group may explain this difference, since McCrary \& Hun ter (1953) have shown that a long intertrial interval accentuates the SPE.

When errors in the experimental group are plotted against the serial position of the items within each trial, a straight line rather than a bowed curve emerges. Slamecka (1967) has said that the SPE appears during both learning and recall. If this were indeed true, both curves in Fig. 2 would be bowed. An experiment similar to this one by Bowman \& Thurlow (1963) also failed to yield a bowed curve. However, they did get an inflected curve, which they called a sine curve, when they plotted errors against the serial position of the items within each trial. They pointed out that this was unexpected, and they were unable to advance a fully convincing explanation. The results obtained in the present study would be expected if the processes of recall did not show a SPE. Bowman and Thurlow's "sine curve" could have resulted from rehearsal during the long $(12-\mathrm{sec})$ intertrial interval. If recall showed a SPE, it would have to show up in Fig. 2, as all other effects, such as those caused by learning, are distributed at random throughout the curve. Thus, the results of this experiment indicate that the SPE is due to processes involved in learning, not recall.

Glanzer \& Dolinsky (1965) have shown that Ss use different items as an anchor in an apparently homogeneous list. Choice of the anchor can be based upon any of several parameters. However, Ss given an anchor use it, and a bowed error curve follows it. In this study an anchor was provided, namely, the numeral " 1 " before the serially first item. The bowed curve found around that item indicates that this technique did indeed provide an anchor.

In the traditional serial learning task, the intertrial interval serves as the anchor, and, consequently, the following item has the fewest errors. This is usually the first item on the list. In the present case, the anchor is the item in the list labelled " 1 ," not the intertrial interval. Therefore, the first item anticipated (i.e., the item following the labelled item) has the fewest errors. Because of this parallel, the item following the anchor item was plotted as the first item in the list in Fig. 1. The anchor item itself was plotted as the last item of the list.

Bowman \& Thurlow (1963) explained the fact that the fewest errors occurred on the second item in this paradigm in terms of differential rates of forwards and backwards learning. Ribback \& Underwood (1950) lend support to this idea, as they showed that backwards learning proceeds more slowly than forwards learning. Although this could explain the fact that the fewest errors fall on the second item, we attribute it to the effect of the anchor, a situation which is similar to having the fewest errors occur on the item following the intertrial in terval.

If, in fact, the intertrial interval does serve as an anchor in the standard paradigm, the anchoring effect must contribute part of the SPE. In this experiment, the anchor produced a quadratic factor about half as great as that in the SPE yielded by the control group. This suggests that the anchoring effect produced by the intertrial interval may contribute about half of the quadratic component in the SPE, or even more if the intertrial interval is a better anchor than the numeral " 1 " used for the experimental group.

The fact that there is a significant difference between the total number of errors made by the two groups indicates that positional cues do play a role in serial learning. That positional cues are not the only cues that can be used is shown by the fact that the experimental group could, in fact, learn the list. This finding is in agreement with that of Battig, Brown, \& Nelson (1963).

\section{REFERENCES}

BATTIG, W. F., BROWN, S. C., \& NELSON, D. Constant vs. varied serial order in pairedassociate learning. Psy chological Reports, 1963 , 12, 695-721.

BOWMAN, R. E., \& THURLOW, W. $R$ Determinants of the effect of position in serial learning. American Journal of Psychology, $1963,73,436-451$. 
DEESE, J., \& KRESSE, F. H. An experimental analysis of the errors in rote serial learning. Jounal of Experimental Psy chology, 1952,44, $199-202$.

GLA2E, J. A. The association value of non-sense syllables. Joumal of Genetic Psychology, 1928, 35, 255-269.

GLANZER, M., \& DOLINSKY, R. The anchor for the serial position curve. Joumal of Verbal Leaming \& Verbal Behavior, 1965, 4, 267-273. GLANZER, M., \& PETERS, S.C. Reexamination of the serial position effect. Journal of Experimental Psychology, 1962, 64, 258-266. HOVLAND, D. I. Experimental studies in rote-learning theory: II. Reminiscence with varying speeds of syllable presentation. Joumal of Experimental Psychology, 1938, 22, 338-353.
MaCRARY, J. W., \& HUNTER, W. S. Serial position curves in verbal learning. Science, 1953,117, 131-134.

RIBBACK, A., \& UNDERWOOD, B. J. An empirical explanation of the skewness of the bowed serial position curve. Joumal of Experimental Psychology, 1950, 40, 329-335. SLAMECKA, N. Human learning and memorySelected readings. New York: Oxford University Press, 1967.

\section{NOTE}

1. We express gratitude to Dr. Walter Makous for his many helpful suggestions, and to Mr. Michael Murray for assistance in the analysis of the data. This study was supported in part by the USPHS Training Grant No. ST01 GM 00666.

\section{Temporal ROC curves and the psychological moment'}

\author{
JONATHAN BARON, Mental Hcalth \\ Research Institute, The University of \\ Michigan, Ann Arbor, Mich. 48104
}

Subjects were asked to tell which of two points on a visual display moved first, and to indicate their confidence according to a six-category scale. One of the two points moved 32 msec before the other in all cases, but the order varied at random. ROC curves were straight lines on normal-normal paper. The results are inconsistent with the theory of a "psychological moment" but are consistent with the idea of "temporal noise" in perception.

Several classes of evidence (Stroud, 1955; White, 1963; Shallice, 1967) point to the existence of a quantal unit of psychological time which has been called the psychological moment. According to this moment theory, psychological time is divided into discrete, nonoverlapping intervals or moments. A specific implication of one form of this theory is that events occurring within the same moment are perceived as simultaneous. Only events falling in different moments are perceived as occurring at different times. The presumed moment should thus limit the ability of Ss to detect the order of occurrence of two stimuli. This limitation would hold, however, only when the $S$ is prevented from using cues other than the apparent times of occurrence of the stimuli, such as apparent motion (Thor, 1968) or apparent position (Békésy, 1967). While estimates of the psychological moment are never less than $50 \mathrm{msec}$, Ss can discriminate the order of two stimuli when their temporal separation is on the order of a millisecond as long as the use of these other cues is permitted.

We shall compare the moment theory to an alternative theory, which we shall call the noise theory. This theory holds that the time between the occurrence of a stimulus and its perception is somewhat variable. Failures to correctly discriminate the order of two stimuli may be ascribed to the variability of the times it takes to perceive the two stimuli. It will be assumed for simplicity, that the distribution of these times is normal.

Kristofferson (1967) has tested the moment theory in an experiment in which Ss were asked to say whether two stimuli were simultaneous or successive. The interval between the stimuli was varied from trial to trial. The critical data was a plot of the probability of saying that the stimuli were successive against the interval between the stimuli. According to the moment hypothesis, such a plot should be fit by a straight line. Although Kristofferson has fit such data with straight lines, others (Hirsch \& Sherrick, 1961) have fit similar data with normal ogives. A normal ogive would be predicted by the noise hypothesis described above. In fact, the two types of curves are difficult to distinguish on the basis of experimental data. An ogive is very close to a straight line over much of its length. Furthermore, if the duration of the moment were only slightly variable, the line predicted by the moment hypothesis would be rounded near probabilities of zero and one, and would look even more like a normal ogive.

The present experiment uses a different approach to distinguish between the moment theory and the noise theory. Like the experiments described above, the $S$ is asked to tell which of two stimuli came first But unlike the above experiments, the temporal separation between the stimuli is held constant, only their order being varied from trial to trial. Also, the $S$ is asked to indicate his confidence in each of his judgments. According to the theory of signal detection (Green \& Swets, 1966) such confidence ratings may be used to infer what the behavior of the $S$ would be if he were asked to vary his criterion or response bias for saying that one of the stimuli came first. The $S$ will be more likely to say that a given stimulus came first when the bias towards that response is high, or when the criterion for it is low. The data of interest to us is a plot of the probability of saying that one of the stimuli came first when it did (hits) against the probability of saying that it came first when it did not (false alarms) as the criterion or response bias is varied. Such a curve is known as the receiver-operatingcharacteristic ( $R O C$ ) curve.

According to the noise hypothesis, the ROC curve should resemble that obtained in other situations in which noise is also presumed to be the limiting factor affecting discriminability. We may apply the theory of signal detection to distributions of perception times for the two stimuli. According to this theory, the confidence ratings are taken to refer to a set of criteria based on a comparison of the probabilities of the two alternatives (one stimulus coming first or the other) given the perceptual experience of the S. The ROC curve should be bowed downwards when plotted in terms of probabilities, but linear when plotted in terms of the normal abscissae corresponding to the probabilities, i.e., on normal-normal coordinates.

According to the moment hypothesis, a "threshold" theory, only when the stimuli fall in different moments will their order be discriminable. Let us call the probability of the two stimuli ( $A$ and $B)$ coming in different moments with one of them (A) coming first, $\mathbf{p}\left(\mathbf{A}^{\prime}\right)$. Let us call the probability of the two stimuli falling within the same moment $\mathrm{p}(\mathrm{M})$. When the stimuli fall within the same moment, it is assumed that the S's guesses are independent of the stimulus conditions and that he has some response bias, $X$, for saying that $S$ timulus $A$ came first. This response bias is reflected in his confidence ratings. Thus the probability of a hit is $p(a / A)=p\left(A^{\prime}\right)+X p(M)$, where $a$ is the $S$ saying that $S$ timulus $A$ came first. On the other hand, the probability of a false alarm is $p(a / B)=X p(M)$. It follows that $p(a / A)=p(a / B)+p\left(A^{\prime}\right)$. Thus the ROC curve predicted by the moment hypothesis is a straight line when plotted in terms of probabilities, and is bowed upwards when plotted on normal-normal paper. Further, the only parameter involved in this equation is $p\left(\mathrm{~A}^{\prime}\right)$, which may be interpreted as the 